\title{
Nonlinear Picosecond-Pulse Propagation through Optical Fibers with Positive Group Velocity Dispersion
}

\author{
Hiroki Nakatsuka, (a) D. Grischkowsky, and A. C. Balant \\ IBM Watson Research Center, Yorktown Heights, New York 10598 \\ (Received 26 May 1981)
}

\begin{abstract}
The predictions of the nonlinear Schrödinger equation have been tested by passing 5.5psec optical pulses through a 70-m single-mode optical fiber. With use of a precise cross correlation technique based on pulse compression, dramatic reshaping of the input pulses into flat-topped, frequency-broadened, and positively chirped 20-psec output pulses with self-steepened fall times of less than 2 psec was observed. The observations are in good agreement with theory.
\end{abstract}

PACS numbers: $42.65 .-\mathrm{k}$

The recent availability of single-mode optical fibers has opened a new era in nonlinear pulse propagation studies. ${ }^{1,2}$ This is due mainly to (1) the small core size (typically $4 \mu \mathrm{m}$ diam) of the fibers, which allows strong nonlinear effects to occur with modest input powers; (2) the well characterized transverse profile of the beam; and (3) the long optical paths. The nonlinear pulse propagation problem breaks into two different aspects according to whether the group velocity dispersion (GVD) of the optical fiber is positive or negative (for typical fused silica fibers, GVD is positive for wavelengths shorter than 1.3 $\mu \mathrm{m}$ and negative for longer wavelengths). For negative GVD, the frequency sweep due to nonlinear self-phase-modulation causes pulse narrowing, while for positive GVD, pulse broadening occurs.

There have been a number of theoretical studies directly relevant to nonlinear pulse propagation in single-mode fibers under a variety of conditions, ${ }^{3-15}$ but there have been few experimental studies. ${ }^{1,2}$ The only previous nonlinear pulsereshaping experiment in single-mode fibers was for the case of negative GVD. This was the recent, well characterized work of Mollenauer, Stolen, and Gordon ${ }^{2}$ in which the pulse narrowing and pulse splitting, characteristic of solitons, were observed.

In this Letter we report the first experimental observations of pulse reshaping and frequency modulation effects for nonlinear pulse propagation in single-mode optical fibers with large positive GVD. We observed that smooth 5.5-psec [full width at half-maximum (FWHM)] input pulses with peak powers of $10 \mathrm{~W}$ were changed by their nonlinear passage through a $70-\mathrm{m}$ fiber into 20-psec flat-topped pulses with self-steepened trailing edges falling off faster than 2 psec, our resolution limit. The output pulses had a broadened frequency spectrum characteristic of linearly chirped, square pulses. We verified that the 20-psec pulses were linearly chirped by compressing them to 1.5 psec, more than 3 times shorter than the input pulse widths. A most important feature of our experimental technique was that we used these 1.5-psec recompressed pulses as probing pulses to measure the output pulse shape from the fiber. The obtained resolution of 2 psec allowed us to compare our observations in detail with a numerical integration of the nonlinear Schrödinger equation.

Our experimental setup is indicated in Fig. 1. The synchronously pumped, mode-locked dye laser and the atomic-sodium-vapor delay line have already been described. ${ }^{16}$ Here, we used 5.5-psec (FWHM) pulses with linewidths less than $2.5 \mathrm{~cm}^{-1}$, close to the transform limit. The input pulse widths to the single-mode optical fiber (manufactured by ITT as Type T-1601 with a core diameter of $4 \mu \mathrm{m}$ ) and the subsequent output pulse widths were measured simultaneously by noncollinear second-harmonic generation in a temperature-tuned ammonium dihydrogen arsenate (ADA) crystal and an angle-tuned potassium dihydrogen phosphate (KDP) crystal. The back-

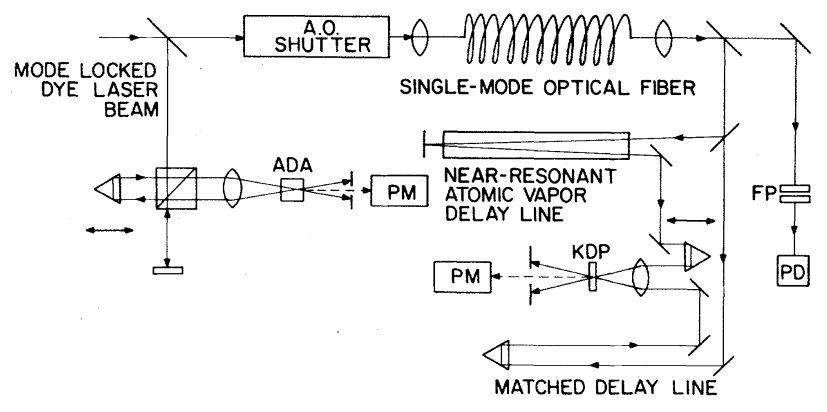

FIG. 1. Schematic diagram of the experiment. 
ward Rayleigh scattering from the fiber was decoupled from the laser by using an acousto-optic shutter to pass single pulses at $4 \mathrm{MHz}$. The optical arrangement for the cross-correlation measurements is shown. We also measured, by autocorrelation, the fiber output pulse widths and the recompressed pulse widths. The peak input power to the fiber was determined from the duty cycle and from the measured average output power from the fiber. To obtain the input and output spectra, we scanned the tilt angle of the Fabry-Perot interferometer while monitoring the transmitted beam.

Our observations are depicted in Fig. 2 for 5874- $\AA$, 10-W, 5.5-psec input pulses to the 70$m$ optical fiber. In Fig. 2(a) the autocorrelated output pulse from the fiber is displayed. The triangular shape is indicative of the square pulse shape, while the sharpness of the peak illustrates that the pulse has detail on $\sim 1$-psec time scale. The positive chirp of this output pulse enabled us to obtain the exceptional compression shown in Fig. 2(b), by passing this pulse through the sodium-vapor recompression cell. ${ }^{16}$ The 20 -psec

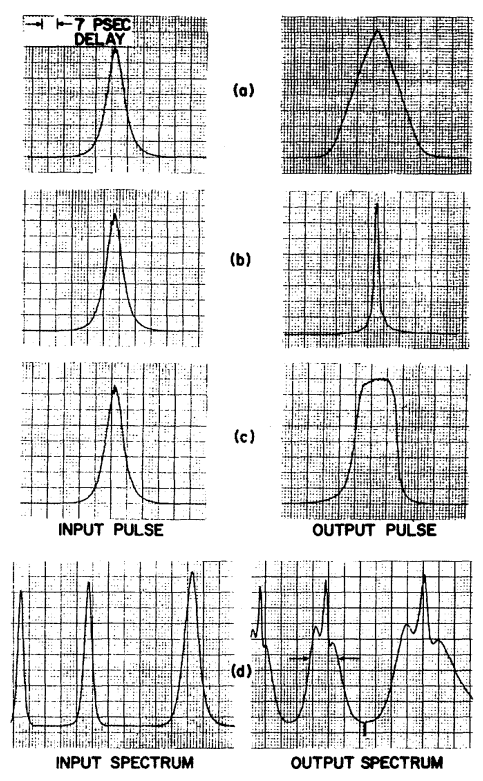

FIG. 2. Input and output pulses and spectra from the $70-\mathrm{m}$ single-mode optical fiber. For all the pulses each large division corresponds to a 7-psec delay. (a) Autocorrelated input and output pulses. (b) Autocorrelated input and recompressed output pulses. (c) Autocorrelated input and cross-correlated output pulses. (d) Input and output Fabry-Perot inteferograms. Spectral range is $25 \mathrm{~cm}^{-1}$; finesse is 100 ; the linewidth indicated by the arrows is $10 \mathrm{~cm}^{-1}$. pulse was compressed to approximately $1.5 \mathrm{psec}$ with a corresponding increase in intensity. This recompressed pulse served as an excellent probing pulse for the cross-correlation measurement of the output pulse, as shown in Fig. 2(c). In addition, the jitter in the repetition rate of the laser was eliminated from the measurement because we cross correlated with the same pulse from the mode-locked train. However, we were still sensitive to the $\pm 0.5-\mathrm{cm}^{-1}$ laser-frequency jitter because of the group velocity dispersion of the recompression cell of approximately $2 \mathrm{psec} /$ $\mathrm{cm}^{-1}$. Therefore we had a timing jitter in our cross correlation of \pm 1 psec. The main features of the cross-correlation measurement of the output pulse are the flat top and the asymmetry between the leading and trailing edges of the pulse. The measured 2-psec drop-off of the trailing edge is our resolution limit. In Fig. 2(d), the input spectrum is seen to be quite clean with a linewidth (FWHM), including frequency jitter, of 2.5 $\mathrm{cm}^{-1}$. The structure of the spectral output from the fiber is characteristic of a linearly chirped, square pulse.

The reduced wave equations describing the propagation of the electric field amplitude $E$ and the phase $\varphi$ through the single-mode fiber are

$$
\begin{aligned}
& \frac{\partial E}{\partial z}+k_{1} \frac{\partial E}{\partial t}=-\frac{k_{2}}{2}\left[2 \frac{\partial \varphi}{\partial t} \frac{\partial E}{\partial t}+E \frac{\partial^{2} \varphi}{\partial t^{2}}\right], \\
& \frac{\partial \varphi}{\partial z}+k_{1} \frac{\partial \varphi}{\partial t}=+\frac{k_{2}}{2}\left[\frac{1}{E} \frac{\partial^{2} E}{\partial t^{2}}-\left(\frac{\partial \varphi}{\partial t}\right)^{2}\right]-\kappa E^{2},
\end{aligned}
$$

where the propagating electric field is given by

$$
E=E \cos \left(k_{0} z-\omega_{0} t-\varphi\right) \text {. }
$$

In Eqs. (1) and (2), $k_{1}=\partial k / \partial \omega=1 / v_{g}$ is the reciprocal of the group velocity $v_{g}$ at the carrier frequency $\omega_{0} ; k_{2}=\partial^{2} k / \partial \omega^{2}$ is the group velocity dispersion at $\omega_{0} ; \kappa=0.5 \alpha k_{0} n_{2} / n_{0}$ (see Ref. 16); $k_{0}$ $=2 \pi n_{0} / \lambda_{0}$ is the propagation vector for the carrier wave with the index of refraction $n_{0}$ and vacuum wavelength $\lambda_{0}$; and $n_{2}$ is the nonlinear index of refraction. With the substitution $u=E \exp (i \varphi)$, these equations are equivalent to the nonlinear Schrödinger equation, ${ }^{2-4,7}$

$$
i\left(\frac{\partial u}{\partial z}+k_{1} \frac{\partial u}{\partial t}\right)=-\frac{1}{2} k_{2} \frac{\partial^{2} u}{\partial t^{2}}+\kappa|u|^{2} u
$$

which also appears in many other branches of physics, e.g., plasma physics, superconductivity, low-temperature physics, water waves, and vortex motion. ${ }^{3,4}$ The derivation of Eq. (3) is based mainly on the slowly varying envelope approxima- 
tion, where the amplitude $E$ and phase $\varphi$ change slowly compared with the oscillation period. Because of the high optical carrier frequency, the physical problem best described by Eq. (3) is that of nonlinear optical pulse propagation in single-mode fibers.

In obtaining the numerical solutions to Eqs. (1) we used the following parameters. The peak input power was $10 \mathrm{~W}$, and $n_{2}$ was $1.1 \times 10^{-13}$ esu. $^{1}$ For convenience, $\alpha$ was set equal to unity. ${ }^{17}$ The group velocity dispersion for our 70-m fiber was measured to be $2.5 \mathrm{psec} / \AA$. The input pulse shape (5.3 psec FWHM) in Fig. 3 is the square of a hyperbolic secant with the addition of the asymmetric wings shown, and agrees well with the measured autocorrelated input pulse. We assumed the pulse to be transform limited.

The results of our calculation for these input conditions are shown in Fig. 3. The most important features of the pulse reshaping are the rapid pulse broadening, the square pulse shape with self-steepened edges, and the positive chirp (not shown) of $13 \mathrm{~cm}^{-1}$ across the $20-$ psec output pulse. ${ }^{18}$

The calculation of Fig. 3 is compared with the experimental results in Fig. 4. The major features are well explained. The calculated peak of the autocorrelation is somewhat rounder than the measurement shown in Fig. 2(a), indicating that the experiment may have a sharper falling edge than the calculation. We believe that the observed

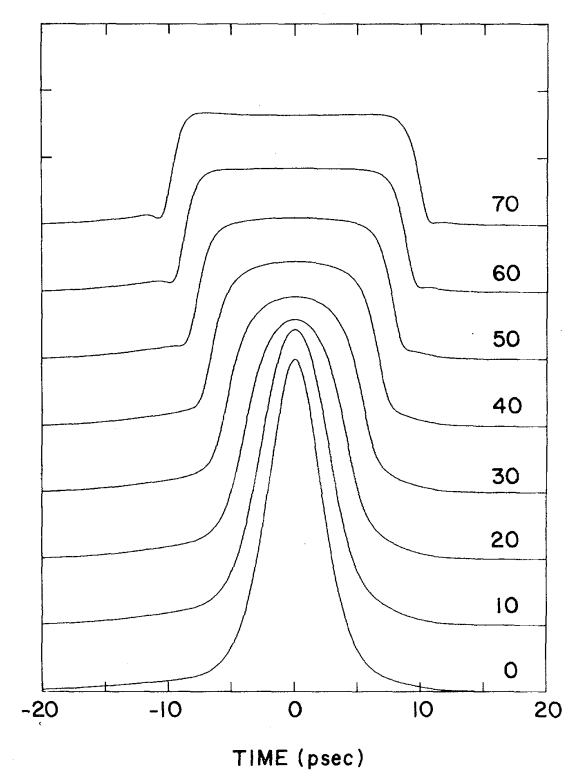

FIG. 3. Calculated pulse shapes vs distance (in meters) propagated in the fiber. wings on the pulses are simply the wings of the input pulses which pass through the fiber undistorted until they are swept up by the expanding edges of the square pulse. Considering the nonlinear nature of this propagation problem, the agreement between theory and experiment is quite good.

A small but perhaps fundamental disagreement is that experimentally the output pulse has a much slower rising edge than the resolutionlimited falling edge. We have tried many calculations with asymmetric input pulses having different rise and fall times, and with and without an initial frequency chirp, but we cannot calculate this feature of our data. Some possible explanations could be the following: (1) an unexpectedly large nonlinear group velocity effect; (2) the nonlinear index may have a small but finite relaxation time; (3) the slowly varying envelope approximation, used to derive the nonlinear Schrödinger equation, may not have sufficient accuracy for long-path, picosecond-pulse propagation; and (4) some effect if the probing pulse were asymmetric.

In conclusion, our work has shown strong and well characterized pulse-reshaping effects in

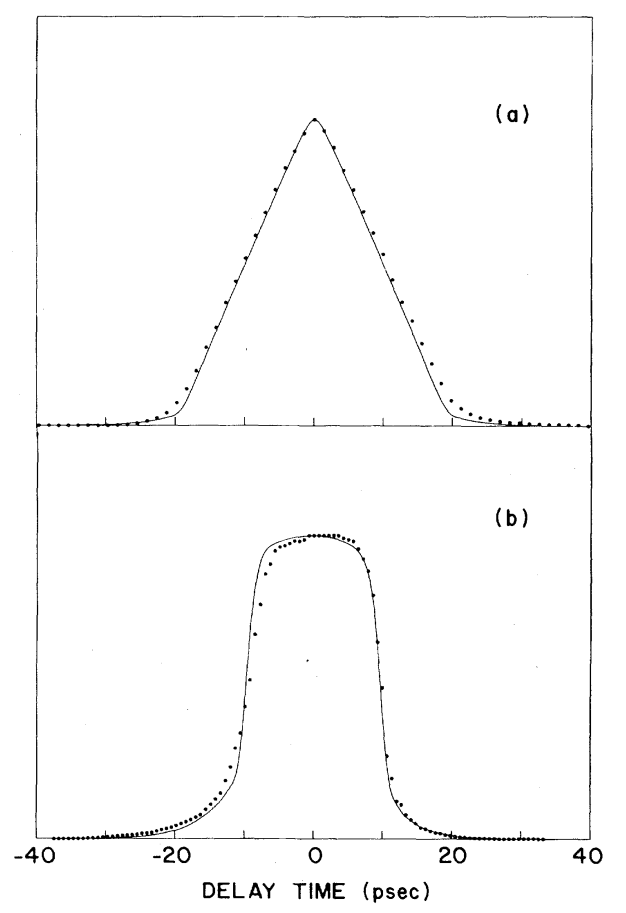

FIG. 4. Comparison between theory (solid line) and experiment (dots). (a) Autocorrelation of the output pulses. (b) Cross correlation. 
single-mode optical fibers with positive GVD. By using a precise cross-correlation scheme, we measured the output pulse shape from the fiber with 2-psec precision and thereby tested both the predictions and the validity of the nonlinear Schrödinger equation.

We would like to acknowledge the careful readings of this manuscript and helpful comments of M. M. T. Loy, Aa. S. Sudbo, and J. J. Wynne. This work was partially supported by the U.S. Office of Naval Research.

\footnotetext{
(a) Permanent address: Department of Physics, Faculty of Science, Kyoto University, Kyoto 606, Japan.

${ }^{1}$ R. H. Stolen and C. Lin, Phys. Rev. A 17, 1448 (1978).

${ }^{2}$ L. F. Mollenauer, R. H. Stolen, and J. P. Gordon, Phys. Rev. Lett. 45, 1095 (1980).

${ }^{3}$ G. B. Whitham, Linear and Nonlinear Waves (Wiley, New York, 1974).

${ }^{4}$ Solitons in Action, edited by K. Lonngren and

A. Scott (Academic, New York, 1978).

${ }^{5}$ V. E. Zakarov and A. B. Shabat, Zh. Eksp. Teor. Fiz. 61, 118 (1972) [Sov. Phys. JETP 34, 62 (1972)], and 64,1627 (1973) [37, $823(1973)]$.

${ }^{6}$ R. H. Stolen and A. Ashkin, Appl. Phys. Lett. 22,
}

294 (1973).

${ }^{7}$ A. Hasegawa and F. Tappert, Appl. Phys. Lett. 23, 142, 171 (1973).

${ }^{8} \mathrm{~J}$. Satsuma and N. Yajima, Prog. Theor. Phys., Suppl. 55, 284 (1974).

${ }^{9}$ R. A. Fisher and W. K. Bischel, J. Appl. Phys. $\underline{46}$, 4921 (1975).

${ }^{10}$ M. Jain and N. Tzoar, J. Appl. Phys. $\underline{49}, 4649$

(1978), and Opt. Lett. $\underline{3}, 202$ (1978).

${ }^{11}$ B. Bendow, P. D. Gianino, N. Tzoar, and J. Jain, J. Opt. Soc. Am. 70, 539 (1980).

${ }^{12}$ B. Bendow and P. D. Gianino, Opt. Lett. $\underline{4}, 164$ (1979).

${ }^{13}$ R. H. Stolen, Proc. IEEE 68, 1232 (1980).

${ }^{14}$ B. Crosignani, C. H. Papas, and P. DiPorto, Opt. Lett. $\underline{6}, 61$ (1981).

${ }^{15}$ N. Tzoar and M. Jain, Phys. Rev. A 23, 1266 (1981).

${ }^{16} \mathrm{H}$. Nakatsuka and D. Grischkowsky, Opt. Lett. $\underline{6}, 13$ (1981).

${ }^{17}$ The constant $\alpha \sim 1$ adapts these equations to describe propagation in single-mode fibers (Refs. 6, 7, 10, 11, and 14).

${ }^{18}$ The general trend of both the data and the calculations as functions of increasing input power is the following. For input power levels below approximately $0.1 \mathrm{~W}$, the pulse is undistorted by passage through the $70-\mathrm{m}$ fiber. As the power is increased above this value, the pulse broadening, chirping, and self-steepening increase monotonically up to our maximum available input power of $10 \mathrm{~W}$.

\title{
Glory in Optical Backscattering from Air Bubbles
}

\author{
Dean S. Langley and Philip L. Marston \\ Department of Physics, Washington State University, Pullman, Washington 99164 \\ (Received 23 December 1980)
}

Observations of light backscattered from air bubbles in a viscous liquid demonstrate an enhancement due to axial focusing. A physical-optics approximation for the crosspolarized scattering correctly describes the spacing of regular features observed. The non-cross polarized scattering is not adequately described by a single class of rays.

PACS numbers: 42.20.Gg, 42.10.Hc, 92.10.Pt

The Mie solution ${ }^{1}$ for electromagnetic scattering by a sphere frequently does not lead to direct interpretation of the angular scattering pattern. Consequently, models have been developed to facilitate an understanding of the structure in the scattered intensity present where intensity is plotted as a function of the scattering angle $\varphi$ or the size parameter $x=k a$ ( $k$ is the wave number; $a$ is the sphere radius). These models have emphasized the angular regions where diffraction is important for a drop of water in air: the rainbow, $,^{2,3} \varphi \simeq 180^{\circ}, 3^{-5}$ and $\varphi \simeq 0^{\circ} .{ }^{3,6}$ In the scattering of light by a spherical air bubble in a liquid or in glass, the real part of the refractive index of the sphere is less than that of the surroundings and the models must be significantly modified. New phenomena appear, such as diffraction ${ }^{7,8}$ in the region of the critical scattering angle $\varphi_{c}$. Here we report the first detailed observations of backscattering by air bubbles in liquids and give a model which describes some of the observed features. We refer to this as glory because, as in the case of drops, ${ }^{3-5}$ the $\varphi \simeq 180^{\circ}$ scattering is enhanced when $x$ is large. 